\section{An agenda for future research projects in polycythemia vera and essential thrombocythemia}

\author{
Tiziano Barbui, ${ }^{1}$ Alessandro Maria Vannucchi, ${ }^{2}$ Paola Guglielmelli, ${ }^{2}$ \\ Valerio De Stefano ${ }^{3}$ and Alessandro Rambaldi ${ }^{4}$
}

${ }^{1}$ FROM Research Foundation, Papa Giovanni XXIII Hospital, Bergamo; ${ }^{2}$ Center of Research and Innovation of Myeloproliferative Neoplasms (CRIMM), Azienda Ospedaliera Universitaria Careggi and Department of Experimental and Clinical Medicine, University of Florence, Florence; ${ }^{3}$ Section of Hematology, Department of Radiological and Hematological Sciences, Catholic University and Fondazione Policlinico Universitario A Gemelli IRCCS, Rome and ${ }^{4}$ Department of Oncology and Hematology, University of Milan, Milan and Azienda Socio Sanitaria Territoriale Papa Giovanni XXIII, Bergamo, Italy

\section{Introduction}

The clinical course of essential thrombocythemia (ET) and polycythemia vera (PV) is characterized by an increased incidence of vascular complications and a tendency to progress to myelofibrosis (MF) or acute myeloid leukemia (AML). Over the past decade, new molecular and clinical knowledge in ET and PV has led to a significant improvement in the diagnostic, prognostic and therapeutic processes. Despite these advancements, many uncertainties remain concerning aspects of clinical decision-making. We identified some unmet needs in clinical practice and research that urgently require new scientific initiatives. For each of these, we reviewed the most significant existing evidence and made proposals for translational and clinical investigations. We acknowledge that several other clinically relevant unmet needs in the management of patients with PV and ET remain. These could not be addressed due to space constrains, and include, above all, prediction of evolution to secondary forms of myelofibrosis, identification of genetic predictors of survival and of specific subgroups of patients to include in intervention trials with novel drugs that are claimed to modify disease course.

\section{Should we look for new diagnostic and prognostic criteria to distiguish} pre-fibrotic myelofibrosis and essential thrombocythemia?

One of the major changes introduced by the 2016 World Health Organization (WHO) classification is the distinction between so called "true" ET and pre-fibrotic PMF. Insights from large series from reference institutions suggest that the proportion of ET patients who would be reclassified as pre-PMF according to these criteria may be as high as $15-30 \%{ }^{1,2}$

Although some clinical and hematologic traits cluster preferentially with prePMF, with the exception of histopathology, no unique distinctive criterion contributes to diagnosis. The same holds true for the mutation landscape, since only a slight increase in CALR mutation frequency is observed in pre-PMF patients. On the other hand, there may be important differences in terms of disease course, clinical complications and overall prognosis. First, the rate of thrombosis seems to be quite similar in pre-PMF and ET ( $2 \%$ patient-years in both conditions). ${ }^{1,3,4}$ Risk variables associated with thrombosis appear to be similar in pre-PMF and ET, as supported by the results of a recent study where the International Prognostic Score of Thrombosis for Essential Thrombocythemia (IPSET-thrombosis) reliably predicted thrombosis in patient with pre-PMF. ${ }^{5}$ In contrast, bleeding episodes may be more frequent in pre-PMF as compared to ET. ${ }^{6}$ Of note, according to large retrospective series, the most distinctive feature between the two diseases is survival, uniformly worse in pre-PMF (ranging from 10.5 to 14.7 years) compared to ET (14.7-21.8 years) in which it was rather similar to the standardized European life expectancy. ${ }^{7,8}$ No specific risk model for survival has yet been developed for pre-PMF; whether that developed for the WHO 2008 definition of ET is proving satisfactory also for pre-PMF remains to be addressed. ${ }^{8}$ The same debate applies also to the recent molecular integrated scores MIPSS-70, that included fibrosis grade 1 versus grade $2 / 3$ (the latter is one distinctive feature of overt-PMF vs. pre$\mathrm{MF}$ ) as a significant variable for survival..$^{9}$ Therefore, since expected survival is the key issue to discuss with a patient newly diagnosed with pre-PMF, acquiring such
Ferrata Storti Foundation
Haematologica 2020
Volume 105(8):1999-2003

\section{Correspondence:}

TIZIANO BARBUI

tbarbui@fondazionefrom.it.

Received: March 4, 2020.

Accepted: April 14, 2020.

Pre-published: May 28, 2020.

doi:10.3324/haematol.2019.246207

Check the online version for the most updated information on this article, online supplements, and information on authorship \& disclosures: www.haematologica.org/content/105/8/1999

\section{(C)2020 Ferrata Storti Foundation}

Material published in Haematologica is covered by copyright. All rights are reserved to the Ferrata Storti Foundation. Use of published material is allowed under the following terms and conditions:

https://creativecommons.org/licenses/by-nc/4.0/legalcode. Copies of published material are allowed for personal or internal use. Sharing published material for non-commercial purposes is subject to the following conditions: https://creativecommons.org/licenses/by-nc/4.0/legalcode, sect. 3. Reproducing and sharing published material for commercial purposes is not allowed without permission in writing from the publisher. 
information definitely represents the most compelling unmet need in pre-PMF. A prospective registry collecting all new cases may be of significant help in finding an answer, but it may take several years. An alternative option may be a study enrolling not only all incident cases, but also retrospective ones, provided all the diagnoses are validated by a centralized panel of expert histopathologists and clinicians. Moreover, a comprehensive clinical and biologic database (with a tissue bank collecting samples at diagnosis and during follow up) should be made available. This approach may help to identify the causes of death in patients with pre-PMF, the rate of transformation to overt PMF and acute leukemia, and possibly allow predictive variables to be identified.

\section{Should patients with essential thrombocythemia or polycythemia vera be stratified in genomic subgroups?}

Recent publications have highlighted the prognostic contribution of genetic information in both ET and PV, which includes driver mutational status, karyotype abnormalities, and presence or absence of mutations in other myeloid genes. Next-generation sequencing (NGS) analysis identified the prognostic relevance of "adverse variants" in terms of inferior overall and shorter leukemia-free or fibrosis-free survival, including ASXL1, SRSF2 and IDH2 in PV and SH2B3, SF3B1, U2AF1, TP53, $I D H 2$, and EZH2 in ET. ${ }^{10}$ Although these studies have certainly contributed to advance our knowledge of the potential prognostic value of mutational genotyping in $\mathrm{PV}$ and ET, and provided informative tools to identify patients at higher risk of disease progression and leukemia transformation, this approach is still far from being considered relevant in clinical practice. In ET, CALR mutations have been shown to correlate with lower risk of thrombosis compared to JAK2V617F mutation and, indeed, the latter is included in the IPSET thrombosis score. ${ }^{11,12}$ In $\mathrm{PV}$, there is some evidence that patients with higher JAK2V617F allele burden may be at increased risk of thrombosis; ${ }^{13}$ however, lack of prospective data weakens the value of this information. Future research should evaluate prospectively whether genetic data may add clinically relevant information on top of a conventional score for PV and the IPSET for ET; not an easy task, when considering that the rate of cardiovascular event is around 2\% patient-years in ET and 2-3\% in $\mathrm{PV}$. Large, international registries may represent the most productive approach using series of patients carefully annotated according to the 2016 WHO classification.

\section{Should cytoreduction be prescribed to all patients with polycythemia vera regardless of risk?}

The first step in approaching a patient with PV is to identify the potential risk of developing major thrombotic or hemorrhagic complications. Patients are considered to be low-risk by age $<60$ years and absence of previous thrombosis ${ }^{14}$ but this distinction is weakening. In fact, low-risk patients optimally treated with phlebotomy and low-dose aspirin still exhibit an annual rate of major thrombotic episodes of $2 \%$ patients/year; an estimate 2 and 3-fold higher than in the general population with, or without, multiple risk-factors, respectively. ${ }^{15}$ Thus, one may argue whether, in the presence of such residual risk of thrombosis, the conservative approach based on phlebotomy and aspirin is still appropriate. The greater added benefit of cytoreductive drugs over phlebotomy in
PV is based on the results of the Polycythemia Vera Study Group 01-PVSG study, ${ }^{16}$ a propensity score analysis of the European Collaboration on Low-dose Aspirin in Polycythemia Vera (ECLAP) $)^{17}$ prospective study and one recent retrospective cohort analysis. ${ }^{18}$ Nonetheless, experts discourage the use of cytoreductive drugs in clinical practice for young patients without previous thrombosis since the supposed leukemogenic risk associated with the currently available drugs, such as hydroxyurea, although largely uncertain, might outweigh the possible antithrombotic benefits.

However, although the prognostic and predictive role of leukocytosis is still debated, it may be worthwhile upgrading low-risk patients presenting with leukocyte counts greater than $11 \times 10^{\circ} / \mathrm{L}$ to the high-risk category and, indeed, these have been included in randomized clinical trials testing hydroxyurea, and ruxolitinib (Mithridate trial; clinicaltrials.gov identifier: NCT4116502). Such a claim could be criticized since there has still been no formal demonstration of a clear advantage of cytoreductive therapy over a well-conducted phlebotomy policy. Therefore, we believe that young patients with no history of previous thrombosis could be exposed to cytoreductive treatment as long as they only receive drugs for which there is no evidence of promoting secondary leukemias or solid tumors. One such a drug is interferon, either in conventional or novel retard formulation, such as Ropeginterferon $\alpha-2 b a$ which has recently been approved by the European Medicines Agency (EMA) for PV based on phase II and III studies. ${ }^{19}$ In these studies, an overall hematologic response in more than $80 \%$ of patients, good tolerability and evidence of molecular responses were found.$^{20}$ The ongoing randomized clinical trial (Low-PV), testing Ropeginterferon $\alpha$ $2 \mathrm{ba}$ in addition to conventional treatment, may hopefully provide a convincing answer to one of the clinical needs of patients with low-risk PV.

Can we improve the prevention and treatment of postmyeloproliferative neoplasms-acute myeloid leukemia?

Leukemia transformation of $\mathrm{PV}$ and $\mathrm{ET}$ is part of the natural history of these diseases. ${ }^{21}$ The risk of leukemic transformation is highest in PMF, but a sizeable proportion of $\mathrm{PV}$ and ET patients are involved, with an estimated risk of $3 \%$ and $1 \%$ at 10 years, respectively..$^{22}$ It is likely that, in these patients, the unrestricted proliferation of bone marrow progenitors lasting for many years may itself favor the leukemic transformation, and, not surprisingly, older age, leukocytosis, and massive thrombocytosis represent significant risk factors in both PV and ET. Most importantly, biologic characteristics such as abnormal karyotype, presence of SRSF2 or IDH2 mutations are emerging risk factors in PV. In ET, anemia, older age, leukocytosis, and presence of TP53 or EZH2 mutations have been reported as risk factors. ${ }^{22}$ Therefore, NGSbased molecular profile performed at diagnosis and during the course of the disease may provide important information to identify patients at higher risk of disease progression and leukemia transformation. ${ }^{10}$

There is no evidence that hydroxyurea, interferon, anagrelide or ruxolitinib can slow down the intrinsic tendency of these diseases to transform into AML. ${ }^{23}$ On the other hand, there are expectations that some new, targeted drugs may reduce the risk of leukemic transformation. Idasanutlin, an MDM2 inhibitor which modulates 
$\mathrm{MDM} 2 / \mathrm{p} 53$ interaction and restores p53 activity, produced clinical responses in PV both as monotherapy and in combination with interferon. ${ }^{24} \mathrm{HDAC}$ inhibitors like givinostat are equally non-genotoxic and active drugs in PV and ET. ${ }^{25}$

As regards the therapeutic approach to post-myeloproliferative neoplasms-acute myeloid leukemia (MPNAML), prognosis is largely dismal except for the few patients who undergo allogeneic hematopoietic stem cell transplantation (alloHSCT), preferably after debulking with induction chemotherapy. However, new drugs are particularly appealing in this setting, including enasidenib and ivosidenib for patients with IDH2 or IDH1 mutated AML, possibly in combination with azacitidine ${ }^{26}$ or ruxolitinib (in case of enasidenib). ${ }^{27}$ In addition, BCL2 inhibitors, like venetoclax or navitoclax, (alone or in combination with hypomethylating agents) and CPX351, a dual-drug liposomal encapsulation of cytarabine and daunorubicin, are also under investigation. At the moment, however, results from rigorously designed clinical trials in this category of high-risk patients are still lacking and the little interest, if any, of pharma companies to promote studies in this setting represents a major problem. It is, therefore, a clear duty of the scientific community to promote academic trials for this unmet clinical need. Since alloHSCT remains the only potentially curative treatment option for these patients, ${ }^{28}$ intensive treatment capable of achieving remission with a full or even incomplete hematologic reconstitution has to be made available. Apart from some new forthcoming protocols, registry collection of the outcomes of these patients is strongly recommended, particularly with the intent to identify biologic subgroups.

\section{Are the current prevention methods for thrombosis adequate?}

The current annual incidence of arterial and venous thrombosis in patients with PV and ET is $2.62 \%$ and $1.77 \%$, respectively, a figure 1.5 -fold and 3.2 -fold higher than that in the general population..$^{12,15-20}$ The antithrombotic role of cytoreductive drugs is uncertain. Hydroxyurea $(\mathrm{HU})$ has demonstrated significant efficacy in preventing arterial thromboses, but doubts remain as to its ability to prevent recurrent venous thromboembolism (VTE), ${ }^{29-31}$ particularly in patients with splanchnic venous thrombosis. ${ }^{32,33}$ The antithrombotic efficacy of interferon- $\alpha$ has not yet been convincingly demonstrated, and the performance of ruxolitinib in PV patients resistant/intolerant to $\mathrm{HU}$ is largely uncertain. ${ }^{34}$ Although there is no direct evidence that thrombocytosis per se is a risk factor for thrombosis, the favorable effect of anagrelide versus $\mathrm{HU}$ in preventing the occurrence of venous thromboembolism in ET, as shown in the PT1 randomized clinical trial, ${ }^{35}$ is of interest. However, the interpretation of that study is complicated by the heterogeneity of the patient population that was diagnosed according to WHO 2008 criteria. Of note, the ANAHYDRET study, that included patients with a diagnosis of ET that strictly followed WHO 2016 criteria, showed non-inferiority in terms of arterial and venous events. ${ }^{36}$ As a whole, there is still no convincing evidence of a clear antithrombotic action of cytoreductive drugs in ET and PV, and new randomized clinical trials with thrombosis as the primary end point are warranted.
The indication of low-dose aspirin (LDA) is mainly based on a phase III trial in $\mathrm{PV}^{37}$ and on retrospective studies in ET, but the quality of evidence is low. ${ }^{38}$ However, new hypotheses are now being tested to improve the antithrombotic efficacy of aspirin in ET. These are based on the notion that accelerated release of new platelets in ET may accelerate the recovery of thromboxane (TX)A2-dependent platelet function during the once-daily (od) LDA dosing interval. Accordingly, inhibition of the surrogate biomarker platelet TXA2 by o.d. LDA is incomplete in $\geq 80 \%$ of ET patients, but it was seen to be improved by a twice-daily regimen. ${ }^{39,40}$ In the phase II ARES randomized trial, ${ }^{41}$ most of the $245 \mathrm{ET}$ patients treated with LDA displayed incomplete platelet inhibition, which was improved by shortening the dosing interval to 12 hours. ${ }^{42}$ The long-term superiority, compliance, and tolerability of an optimized LDA regimen is now being investigated in a clinical trial.

The prevention of recurrent venous thromboembolism by vitamin K-antagonists (VKA) was estimated in several retrospective studies showing an annual incidence of VTE recurrences as high as 5.6-6.5, ${ }^{31,43-46}$ that rose to as high as 12.8 after discontinuation. ${ }^{45}$ In addition, the incidence of major bleeding on VKA of $1.7-1.8$ per 100 patients/years ${ }^{44,45}$ is unsatisfactory when compared with non-MPN patients. As mentioned above, the addition of hydroxyurea to VKA has a weak effect ${ }^{31}$ and the combined treatment with LDA significantly increases the risk of bleeding with no substantial benefit on antithrombotic prevention. ${ }^{43,47}$ Thus, the prevention of recurrences after VTE is a crucial unmet clinical need in MPN, and innovative strategies are needed. Direct oral anticoagulants (DOAC) can represent a suitable alternative, but before embarking on a formal randomized comparative trial assessing DOAC versus warfarin, a retrospective analysis of treated cases may guide its design. In this regard, some preliminary evidence has been presented in the prospective multicenter observational REVEAL study. $^{47}$

\section{Conclusion}

We have highlighted some clinical topics about which the available evidence is limited. We believe these areas represent priorities for future research projects. Since both PV and ET are relatively rare diseases, and the outcomes of interest occur after long periods of observation, the methodology to conduct these studies cannot be reasonably based on conventional phase II/III design. Therefore, well organized observational and registrybased studies will play a key role in analyzing the clinical outcomes, hopefully with the help of a data mining approach and artificial intelligence techniques, as suggested by preliminary experiences in patients with PV treated with ruxolitinib. ${ }^{48}$

\section{Acknowledgments}

This study was supported by Fondazione per la Ricerca Ospedale (FROM), Papa Giovanni XXIII Hospital, Bergamo, Italy; and by Associazione Italiana per la Ricerca sul Cancro, Grant 5 per Mille, Progetto MYNERVA (P.G. and A.M.V.) and Progetto ISM (AR) 


\section{References}

1. Guglielmelli P, Pacilli A, Rotunno G, et al. Presentation and outcome of patients with 2016 WHO diagnosis of prefibrotic and overt primary myelofibrosis. Blood. 2017;129(24):3227-3236

2. Rumi E, Boveri E, Bellini M, et al. Clinical course and outcome of essential thrombocythemia and prefibrotic myelofibrosis according to the revised WHO 2016 diagnostic criteria. Oncotarget. 2017;8(60): 101735-101744

3. Carobbio A, Finazzi G, Guerini V, et al. Leukocytosis is a risk factor for thrombosis in essential thrombocythemia: Interaction with treatment, standard risk factors, and Jak2 mutation status. Blood. 2007;109 (6):2310-2313

4. Carobbio A, Thiele J, Passamonti F, et al. Risk factors for arterial and venous thrombosis in WHO-defined essential thrombocythemia: An international study of 891 patients. Blood. 2011:117(22):5857-5859.

5. Guglielmelli P, Carobbio A, Rumi E, et al. Validation of the IPSET score for thrombosis in patients with prefibrotic myelofibrosis. Blood Cancer J. 2020;10(2):21.

6. Finazzi G, Vannucchi AM, Barbui T. Prefibrotic myelofibrosis: treatment algorithm 2018. Blood Cancer J. 2018;8(11):104.

7. Barbui T, Thiele I, Passamonti F, et al. Survival and disease progression in essential thrombocythemia are significantly influenced by accurate morphologic diagnosis: A international study. J Clin Oncol. 2011;29 (23):3179-3184.

8. Tefferi A, Guglielmelli P, Larson DR, et al. Long-term survival and blast transformation in molecularly annotated essential thrombocythemia, polycythemia vera, and myelofibrosis. Blood. 2014;124(16):25072513.

9. Guglielmelli P, Lasho TL, Rotunno G, et al. MIPSS70: Mutation-enhanced international prognostic score system for transplantation-age patients with primary myelofibrosis. J Clin Oncol. 2018;36(4):310-318.

10. Tefferi A, Guglielmelli P, Lasho TL, et al. Mutation-enhanced international prognostic systems for essential thrombocythaemia and polycythaemia vera. $\mathrm{Br} \mathrm{J}$ Haematol. 2020 Jan 16. [Epub ahead of print].

11. Barbui T, Vannucchi AM, Buxhofer-Ausch $\mathrm{V}$, et al. Practice-relevant revision of IPSETthrombosis based on 1019 patients with WHO-defined essential thrombocythemia. Blood Cancer J. 2015;5:e369.

12. Barbui T, Finazzi G, Carobbio A, et al. Development and validation of an International Prognostic Score of thrombosis in World Health Organization-essential thrombocythemia (IPSET-thrombosis). Blood. 2012:120(26):5128-5133

13. Vannucchi AM, Antonioli E, Guglielmelli P, et al. Clinical profile of homozygous JAK2 $617 \mathrm{~V}>\mathrm{F}$ mutation in patients with polycythemia vera or essential thrombocythemia. Blood. 2007;110(3):840-846.

14. Barbui T, Tefferi A, Vannucchi AM, et al. Philadelphia chromosome-negative classical myeloproliferative neoplasms: Revised management recommendations from European LeukemiaNet. Leukemia. 2018;32(5):1057-1069.

15. Barbui T, Carobbio A, Rumi E, et al. To the editor: In contemporary patients with polycythemia vera, rates of thrombosis and risk factors delineate a new clinical epidemiology. Blood. 2014;124(19):30213023.

16. Berk PD, Goldberg JD, Donovan PB, Fruchtman SM, Berlin NI, Wasserman LR. Therapeutic recommendations in polycythemia vera based on Polycythemia Vera Study Group protocols. Semin Hematol. 1986;23(2):132-143.

17. Barbui T, Vannucchi AM, Finazzi G, et al. A reappraisal of the benefit-risk profile of hydroxyurea in polycythemia vera: A propensity-matched study. Am J Hematol. 2017:92(11):1131-1136.

18. Enblom-Larsson A, Girodon F, Bak M, et al. A retrospective analysis of the impact of treatments and blood counts on survival and the risk of vascular events during the course of polycythaemia vera. $\mathrm{Br} \mathrm{J}$ Haematol. 2017;177(5):800-805.

19. Gisslinger H, Zagrijtschuk O, BuxhoferAusch V, et al. Ropeginterferon alfa-2b, a novel IFN $\alpha-2 b$, induces high response rates with low toxicity in patients with polycythemia vera. Blood. 2015;126(15):17621769.

20. Gisslinger H, Klade C, Georgiev P, et al. Ropeginterferon alfa- $2 b$ versus standard therapy for polycythaemia vera (PROUDPV and CONTINUATION-PV): a randomised, non-inferiority, phase 3 trial and its extension study. Lancet Haematol. 2020;7(3):196-e208.

21. Tam CS, Nussenzveig RM, Popat U, et al. The natural history and treatment outcome of blast phase BCR-ABL - myeloproliferative neoplasms. Blood. 2008;112(5):1628-1637.

22. Tefferi A, Mudireddy M, Mannelli F, et al. Blast phase myeloproliferative neoplasm: Mayo-AGIMM study of 410 patients from two separate cohorts. Leukemia. 2018;32(5) :1200-1210.

23. Björkholm M, Derolf ÅR, Hultcrantz M, et al. Treatment-related risk factors for transformation to acute myeloid leukemia and myelodysplastic syndromes in myeloproliferative neoplasms. J Clin Oncol. 2011;29 (17):2410-2415.

24. Mascarenhas J, Lu M, Kosiorek H, et al. Oral idasanutlin in patients with polycythemia vera. Blood. 2019;134(6):525533.

25. Rambaldi A, Iurlo A, Vannucchi AM, et al. Safety and efficacy of the maximum tolerated dose of givinostat in polycythemia vera: a two-part Phase Ib/II study. Leukemia. 2020 Feb 11. [Epub ahead of print].

26. Odenike O. How I treat the blast phase of Philadelphia chromosome-negative myeloproliferative neoplasms. Blood. 2018;132(22):2339-2350.

27. McKenney AS, Lau AN, Somasundara $\mathrm{AVH}$, et al. JAK2/IDH-mutant-driven myeloproliferative neoplasm is sensitive to combined targeted inhibition. J Clin Invest. 2018; 128(2):789-804.

28. Lancet JE, Uy GL, Cortes JE, et al. Cpx-351 (cytarabine and daunorubicin) liposome for injection versus conventional cytarabine plus daunorubicin in older patients with newly diagnosed secondary acute myeloid leukemia. J Clin Oncol. 2018;36(26):2684-2692.

29. Barbui T, De Stefano V, Ghirardi A, Masciulli A, Finazzi G, Vannucchi AM. Different effect of hydroxyurea and phlebotomy on prevention of arterial and venous thrombosis in Polycythemia Vera. Blood Cancer J. 2018;8(12):124.
30. De Stefano V, Carobbio A, Di Lazzaro V, et al. Benefit-risk profile of cytoreductive drugs along with antiplatelet and antithrombotic therapy after transient ischemic attack or ischemic stroke in myeloproliferative neoplasms. Blood Cancer J. 2018;8(3):25.

31. De Stefano V, Rossi E, Carobbio A, et al Hydroxyurea prevents arterial and late venous thrombotic recurrences in patients with myeloproliferative neoplasms but fails in the splanchnic venous district. Pooled analysis of 1500 cases. Blood Cancer J. 2018;8(11):112.

32. De Stefano V, Vannucchi AM, Ruggeri M, et al. Splanchnic vein thrombosis in myeloproliferative neoplasms: Risk factors for recurrences in a cohort of 181 patients. Blood Cancer J. 2016;6(11):e493.

33. Sant'Antonio E, Guglielmelli P, Pieri L, et al. Splanchnic vein thromboses associated with myeloproliferative neoplasms: An international, retrospective study on 518 cases. Am J Hematol. 2020;95(2):156-166.

34. Masciulli A, Ferrari A, Carobbio A, Ghirardi A, Barbui T. Ruxolitinib for the prevention of thrombosis in polycythemia vera: a systematic review and meta-analysis. Blood Adv. 2020;4(2):380-386.

35. Harrison CN, Campbell PJ, Buck G, et al. Hydroxyurea compared with anagrelide in high-risk essential thrombocythemia. N Engl J Med. 2005;353(1):33-45.

36. Gisslinger $\mathrm{H}$, Gotic $\mathrm{M}$, Holowiecki J, et al. Anagrelide compared with hydroxyurea in WHO-classified essential thrombocythemia: the ANAHYDRET Study, a randomized controlled trial. Blood. 2013;121(10):1720-1728.

37. Landolfi R, Marchioli R, Kutti J, et al. Efficacy and Safety of Low-Dose Aspirin in Polycythemia Vera. N Engl J Med. 2004;350 (2):114-124.

38. Chu DK, Hillis CM, Leong DP, Anand SS, Siegal DM. Benefits and risks of antithrombotic therapy in essential thrombocythemia: A systematic review. Ann Intern Med. 2017;167(3):170-180

39. Pascale S, Petrucci G, Dragani A, et al. Aspirin-insensitive thromboxane biosynthesis in essential thrombocythemia is explained by accelerated renewal of the drug target. Blood. 2012;119(15):35953603 .

40. Dillinger JG, Sollier CBD, Sideris G, Ronez E, Henry P, Drouet L. Twice daily aspirin to improve biological aspirin efficacy in patients with essential thrombocythemia. Thromb Res. 2012;129(1):91-94.

41. De Stefano V, Rocca B, Tosetto A, et al The Aspirin Regimens in Essential Thrombocythemia (ARES) phase II randomized trial design: Implementation of the serum thromboxane B2 assay as an evaluation tool of different aspirin dosing regimens in the clinical setting. Blood Cancer J. 2018;8(6):49.

42. Rocca B, Tosetto A, Betti S, et al. A randomized, double-blind trial of three aspirin regimens to optimize antiplatelet therapy in essential thrombocythemia. Blood. 2020 april 7. [Epub ahead of print]

43. De Stefano V, Za T, Rossi E, et al. Recurrent thrombosis in patients with polycythemia vera and essential thrombocythemia: Incidence, risk factors, and effect of treatments. Haematologica. 2008;93(3):372-380.

44. Hernández-Boluda J-C, Arellano-Rodrigo E, Cervantes F, et al. Oral anticoagulation to prevent thrombosis recurrence in poly- 
cythemia vera and essential thrombocythemia. Ann Hematol. 2015;94(6):911918.

45. De Stefano V, Ruggeri M, Cervantes F, et al. High rate of recurrent venous thromboembolism in patients with myeloproliferative neoplasms and effect of prophylaxis with Vitamin $\mathrm{K}$ antagonists. Leukemia. 2016;30(10):2032-2038.

46. Wille K, Sadjadian P, Becker T, et al. High risk of recurrent venous thromboembolism in BCR-ABL-negative myeloproliferative neoplasms after termination of anticoagulation. Ann Hematol. 2019;98(1):93-100.

47. Zwicker JI, Lessen DS, Colucci P Paranagama D, Grunwald MR. Risk of hemorrhage in patients with polycythemia vera exposed to aspirin in combination with anticoagulants: results of a prospective, multicenter, observational cohort study (REVEAL). Blood. 2019;134 (Supplement 1):168.

48. Verstovsek S, De Stefano V, Heidel FH, et al. US Optum Database study in polycythemia vera patients: thromboembolic events (TEs) with hydroxyurea (HU) vs ruxolitinib switch therapy and machinelearning model to predict incidence of TEs and HU failure. Blood. 2019;134 (Supplement 1):1659. 